\section{Estresse de desmame influencia a resposta in vitro de fator de necrose tumoral em potros Mangalarga Marchador}

\author{
Weaning stress influences the in vitro response of the tumor \\ necrosis factor in Mangalarga Marchador foals
}

\author{
Bruno Ferreira Spindola*, Paulo de Tarso Landgraf Botteon², Fabio Fagundes Rocha ${ }^{3}$, Simone Bizerra Calado4, \\ Rita de Cassia Campbell Machado Botteon², Isabella Manes Souto Mayor da Mota Rodrigues \& \\ Lucas Moura Marinho ${ }^{5}$ \\ 'Médicos veterinários, DR. Hospital Veterinário, Instituto de Veterinária, Universidade Federal Rural do Rio de Janeiro - UFRRJ, \\ Seropédica, RJ, Brasil \\ 2Médicos veterinários, Dr, Professores. Departamento de Clínica e Cirurgia Veterinária, Instituto de Veterinária, Universidade \\ Federal Rural do Rio de Janeiro - UFRRJ, Seropédica, RJ, Brasil \\ ${ }^{3}$ Médico veterinário, PhD, Professor. Departamento de Ciências Fisiológicas, Instituto de Biologia, Universidade Federal Rural do \\ Rio de Janeiro - UFRRJ, Seropédica, RJ, Brasil \\ ${ }^{4}$ Médica veterinária, MSc. Hospital Veterinário, Universidade Federal Rural do Rio de Janeiro - UFRRJ, Seropédica, RJ, Brasil \\ ${ }^{5}$ Médico veterinário. Universidade Federal Rural do Rio de Janeiro - UFRRJ, Seropédica, RJ, Brasil
}

\section{Resumo}

O desmame precoce é um período crítico para uma saúde e o desenvolvimento de potros, devido a uma queda de resistência imunológica (imonocompetência) tornando o potro mais susceptível a doenças e infecções. O presente estudo objetivou avaliar as pesquisas provocadas por manejo de desmame abrupto sobre uma concentração plasmática de cortisol total e uma ocorrência de imunossupressão celular, medida pela expressão do fator de necrose tumoral alfa (TNF- $\alpha$ ). O desmame abrupto foi realizado em oito potros Mangalarga Marchador com idades entre 24 e 28 semanas. Os dicionários são dois dias antes do desmame (DAD), e um, sete e 15 dias após o desmame (DApD). Nestes momentos, foram colhidas amostras de sangue total para determinação da concentração plasmática de cortisol total e uma segunda alíquota foi empregada na avaliação da produção de TNF- $\alpha$, mediante o tratamento in vitro com lipopolissacarídeos (LPS) e dexametasona (Dx), de modo a demonstrar se o estresse do desmame produz efeitos similares ao glicocorticoide exógeno sobre a expressão da citocina TNF- $\alpha$. As amostras de sangue total foram fracionadas em outras três alíquotas de $900 \mu \mathrm{L}$. Estas amostras foram tratadas com LPS de Escherichia coli ou Dx e uma alíquota para mantida como controle (CTL). Houve aumento da concentração de cortisol total 24 horas após o desmame (1 DApD) (P<0,0001), momento em que uma expressão in vitro TNF- $\alpha$ apresentou o menor valor nenhum grupo LPS, Grupo ao grupo controle e grupo Dx. Aos 15 DApD, a resposta in vitro de TNF- $\alpha$ foi significativamente maior sem grupo LPS (P = 0,0006). Concluiu-se que o desmame provoca uma condição de estresse agudo nos potros, que resultam em redução da imunidade mediada por células, expressa pela redução dos níveis de TNF- $\alpha$.

Palavras-chave: cortisol, imunidade celular, potro, estresse, TNF- $\alpha$.

\begin{abstract}
This study aimed to evaluate the changes caused by the abrupt weaning of plasma total cortisol and the occurrence of cell immunosuppression, measured by the expression of tumor necrosis factor alpha (TNF- $\alpha$ ). The abrupt weaning was performed in eight Mangalarga Marchador foals aged between 24 and 28 weeks. The animals were evaluated in four moments: seven days before weaning (DAD), and one day after weaning (DApD), seven DApD and 15 DApD. In these moments, blood samples were taken for determination of total plasma cortisol and the expression in vitro of TNF- $\alpha$ treated with lipopolysaccharide from $E$. coli (LPS) or dexamethasone (Dx). There was an increase of total plasma cortisol 24 hours after weaning (1 DAPD) $(\mathrm{P}<0.0001)$, time that the expression in vitro of TNF- $\alpha$ showed the lowest value in the LPS group, similar to the control group and the Dx group. After 15 DAPD, in vitro response of TNF- $\alpha$ was significantly higher in the LPS group ( $\mathrm{P}=0.0006$ ). It was concluded that weaning stress provokes an acute condition in foals, which resulted in a decrease of cell-mediated immunity, expressed by lower TNF- $\alpha$ levels.
\end{abstract}

Keywords: cortisol, cellular immunity, foal, stress, TNF- $\alpha$. \section{BJ M \\ Brazilian Journal of Veterinary Medicine \\ p-ISSN 0100-2430 \\ e-ISSN 2527-2179 \\ ○}

Como citar: Spindola, B. F., Botteon, P. T. L., Rocha, F. F., Calado, S. B., Botteon, R. C. C. M., Rodrigues, I. M. S. M. M., \& Marinho, L. M. (2017). Estresse de desmame influencia a resposta in vitro de fator de necrose tumoral em potros Mangalarga Marchador. Brazilian Journal of Veterinary Medicine, 39(3), 165-169. doi: 10.29374/2527-2179.bjvm016316

Fonte de financiamento: Coordenação de Aperfeiçoamento de Pessoal de Nível Superior (CAPES), pela concessão de bolsa de mestrado, e Fundação de Apoio à Pesquisa do Estado do Rio de Janeiro (Faperj) pelo apoio financeiro ao projeto.

Comissão de ética: Projeto aprovado pela CEUA-Fapur/UFRRJ - em 04/01/2013.

Conflito de interesses: Os autores declaram não haver conflito de interesses que precisam ser informados

Recebido: Outubro 31, 2016.

Aceito: Maio 15, 2017.

O estudo foi realizado no campus da Universidade Federal do Rio de Janeiro - UFRRJ, Seropédica, RJ, Brasil.

\section{*Correspondência}

Bruno Ferreira Spindola

Instituto de Veterinária, Universidade Federal Rural do Rio de Janeiro - UFRRJ

Rod. BR 465, Km 7

CEP 23851-890 - Seropédica (RJ), Brasil

E-mail: bfspindolla@yahoo.com.br

Copyright Spindola et al. Este é um artigo publicado em acesso aberto (Open Access) sob a licença Creative Commons Attribution Non-Commercial, que permite uso, distribuição e reprodução em qualque meio, sem restrições desde que sem fins comerciais e que o trabalho original seja corretamente citado. 


\section{Introdução}

Estresse foi definido como qualquer evento que provoque aumento da atividade do eixo hipotálamo-hipófise-adrenal (Harbuz \& Lightman, 1992), resultando na elevação da concentração plasmática de cortisol. No equino, estresse foi relacionados a cólicas, fraturas, transporte exercícios (Stull et al., 2004). O estresse induzido por exercícios foi capaz de alterar a capacidade de resposta imune mediada por células em equinos (Cywinska et al., 2013). No desmame de potros foram relacionados como fatores estressantes a privação materna, alterações na habitação, alimentação e estrutura social (Hoffman et al., 1995; Malinowski et al., 1990; Turner et al., 2003). Em potros tem sido relatado aumento da susceptibilidade a uma variedade de infecções respiratórias e gastrointestinais, principalmente as causadas por patógenos intracelulares, em decorrência do estresse de desmame (Page et al., 2011), um provável reflexo do comprometimento da resposta imune mediada por células durante o processo de desmame. No desmame de potros foram relacionados como fatores estressantes a privação materna, alterações na habitação, alimentação e estrutura social, (Hoffman et al.,1995; Malinowski et al.,1990; Turner et al., 2003). Morel (2003) relacionou quatro diferentes formas de manejo do desmame: abrupta, gradual, em pares e em piquetes. No desmame abrupto separa-se a égua e o potro de forma repentina e não existe contato entre eles após a separação. O desmame em pares é realizado de forma abrupta com dois potros da mesma idade em uma mesma baia. O desmame gradual é realizado aumentando o tempo de separação entre o potro e a égua ao longo de semanas. Em condições naturais o potro será desmamado aos nove ou dez meses de idade (Mills \& Nankervis, 2005; Morel, 2005; Schweizer et al., 2006). Em fazendas de criação intensiva, os potros são desmamados com idade variando entre cinco a sete meses, visando reduzir o esforço de éguas gestantes; evitar o comprometimento dos aprumos, em potros mais desenvolvidos, pela posição adotada no ato de mamar; ou poupar éguas idosas e desgastadas (Schweizer et al., 2006; Morel, 2005).

Este estudo teve como objetivo verificar a influência do desmame abrupto sobre a concentração plasmática de cortisol e a expressão da citocina inflamatória fator de necrose tumoral- $\alpha$ (TNF $\alpha$ ), reconhecida como participante da resposta imunee de doenças inflamatórias, de modo a verificar como a TNF- $\alpha$ é afetada pela secreção de glicocorticoides endógenos.

\section{Materiais e métodos}

Foram avaliados oito potros da raça Mangalarga Marchador com idade entre 24 e 28 semanas, seis machos e duas fêmeas, procedentes do criatório da Universidade Federal Rural do Rio de Janeiro. O desmame foi realizado de forma abrupta e simultânea para todos os potros avaliados, que foram conduzidos a um piquete na companhia de uma única égua (madrinha), sem nenhum parentesco com os animais desmamados.

Amostras de sangue coletadas por venopunção jugular a vácuo foram obtidas em tubos heparinizados em quatro momentos: sete dias antes do desmame (7DAD), um dia após o desmame (1DApD), e novamente sete e 15 dias após o desmame (7 DApD e 15 DApD). Estas amostras foram acondicionadas em gelo e transportadas ao Laboratório de Pesquisas Clínicas em Grandes Animais da UFRRJ, onde foram imediatamente processadas.

As amostras foram fracionadas, sendo a primeira parte para obtenção direta de plasma, visando análise da concentração plasmática de cortisol, determinado por radioimunoensaio. A segunda parte, foi empregada na avaliação da produção de TNF- $\alpha$, mediante o tratamento in vitro com lipopolissacarídeos e glicocorticoide, de modo a demonstrar se o estresse do desmame produz efeitos similares ao glicocorticoide exógeno sobre a expressão da citocina TNF- $\alpha$. Para tanto, as amostras de sangue total foram fracionadas em outras três alíquotas de $900 \mu \mathrm{L}$ e submetidas ao teste de estimulação de citocinas in vitro. As alíquotas foram submetidas aos seguintes tratamentos in vitro: 1- Controle negativo (CTL): $900 \mu \mathrm{L}$ de sangue e $100 \mu \mathrm{L}$ de tampão fosfato; 2- DxLPS: $900 \mu \mathrm{L}$ de sangue adicionada de $50 \mu \mathrm{L}$ de Lipopolissacarídeos (LPS) obtido de Escherichia coli (2880, Sigma) 30ng/ml e $50 \mu \mathrm{L}$ de dexametasona $10^{-7} \mathrm{M}$ (Dexametasona 21-fosfato, D1159, Sigma); 3- LPS: $900 \mu \mathrm{L}$ de sangue, $50 \mu \mathrm{L}$ de tampão fosfato (Controle positivo) e $50 \mu \mathrm{L}$ de LPS 30ng $/ \mathrm{ml}$. Estas alíquotas (CTL; DxLPS e LPS) foram incubadas em câmara úmida a $37^{\circ} \mathrm{C}$ a $5 \%$ de $\mathrm{CO}_{2}$, por 16 horas; após isto, foram centrifugadas por 10 minutos a 15.000 g e o plasma coletado foi analisado para determinação da concentração de fator de necrose tumoral 
alfa (TNF- $\alpha$ ) pelo método ELISA (kit de ELISA para equino (Equus caballus) da Uscn Life Science Inc) a 450 nm, conforme descrito por DeRijk et al. (1997).

Os valores das concentrações plasmáticas de cortisol e TNF- $\alpha$ foram analisados pelo teste anova para medidas repetidas, e teste de Tukey como pós-teste, ao nível de significância de 5\%.

\section{Resultados e discussão}

O potencial do desmame abrupto em promover estresse tem sido demonstrado em leitões, bezerros e potros (Malinowski et al., 1990; Hoffman et al.,1995; Juul-Madsen et al., 2010). Contudo, a resposta celular decorrente deste processo, ainda é pouco documentada. Neste estudo, ocorreu elevação significativa $(\mathrm{P}<0,0001)$ da concentração de plasmática de cortisol no primeiro dia após o desmame (1 DApD). Os valores basais registrados sete dias antes do desmame (7 DAD) foram de 23,85 ng/mL, elevaram-se a 43,80ng/mL1 DApD e retornaram a valores semelhantes ao basal aos 7 DApD 23,13ng/mL e 14 DApD 18,46ng/mL (Figura 1). Estes dados foram semelhantes aos observados por Houpt et al. (1984), Hoffman et al. (1995), Malinowski et al. (1990) e Turner et al. (2003), concordando que o modelo de desmame empregado neste experimento é um manejo estressante para os potros.

Potros sob condição de estresse de desmame, apresentam redução da proliferação de linfócitos (Malinowski et al., 1990; Turner et al., 2003). Segundo Chrousos (1995), a elevação das concentrações de plasmáticas de cortisol ou glicocorticoides suprime a produção de TNF- $\alpha$, interferon gama (IFN- $\gamma$ ) e interleucina (IL) 2 in vitro e in vivo tanto em humanos como em animais. Adams \& Horohov (2013) evidenciaram a redução das concentrações das citocinas (IFN- $\gamma$, TNF- $\alpha$ e IL-10) em pôneis submetidos a tratamento com imunomodulador após desmame.

Neste estudo, verificou-se um aumento significativo ( $\mathrm{P}=0.0006)$ da expressão do TNF- $\alpha$, somente no momento 15 DApD, no grupo LPS, não havendo nos demais momentos diferença significativa entre os tratamentos (Figura 2). A análise destes dados nos permite inferir que houve inibição da expressão do TNF- $\alpha$ no grupo LPS, nos momentos 1DApD e 7 DApD, provavelmente em decorrência da concentração de cortisol endógeno. A expressão do TNF- $\alpha$ no grupo LPS foi inversa à concentração de cortisol plasmático nos potros. Aos 15 DApD, com a redução do cortisol, houve elevação da expressão do TNF- $\alpha$, indicando que o estresse decorrente da desmama produziu cortisol endógeno suficiente para suprimir a resposta imune celular.

OTNF- $\alpha$ é uma citocina pró-inflamatória tipo 1, responsável pelo resposta imune mediada por células como macrófagos e monócitos, que iniciam uma resposta imune àinfecção, particularmente importante na eliminação de infecções bacterianas (Bemelmans et al., 1996; Kim \& Maes, 2003). O desafio com LPS mimetiza esta condição e estimula a produção de TNF- $\alpha$. Os sete primeiros dias de separação do potro expõem estes animais a infecções oportunista, dada à redução da

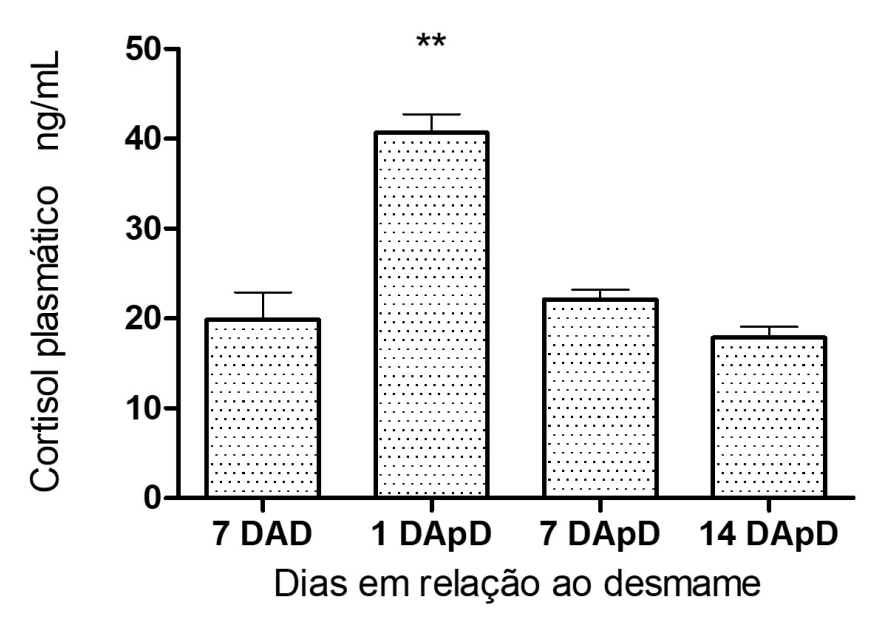

Figura 1. Concentrações plasmáticas de cortisol, em ng/mL, de potros submetidos ao desmame precoce abrupto, nos momentos sete dias antes do desmame (DAD) e um, sete e 15 dias após o desmame (DApD), com a presença de égua madrinha. ${ }^{* *}$ Diferença significativa (valor $\mathrm{p}=0,0001$ ). 


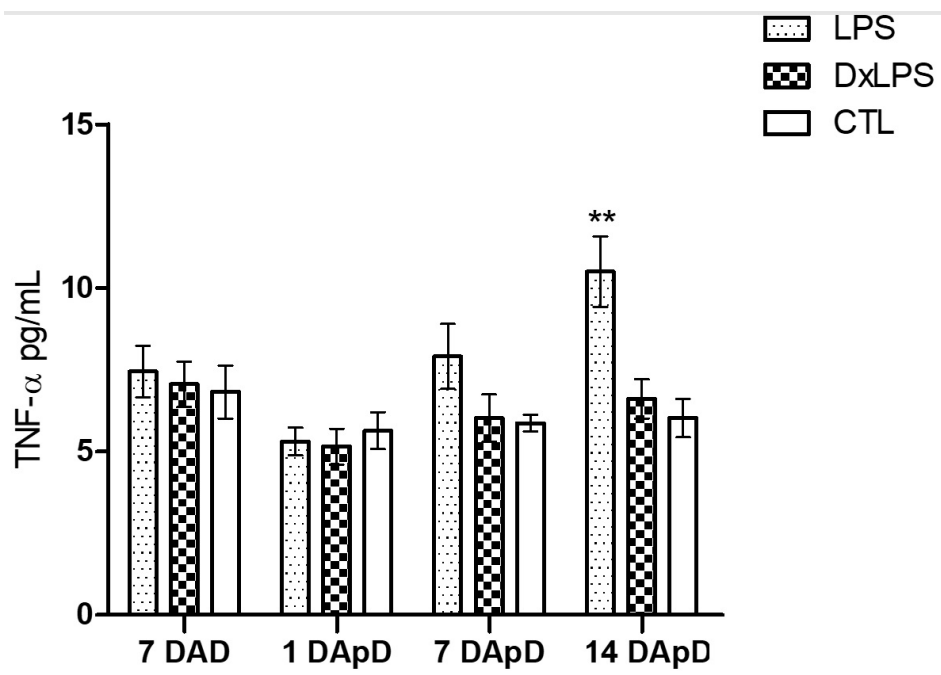

Figura 2. Expressão de TNF- $\alpha$ in vitro, em amostras de sangue de potros sete dias antes do desmame (DAD) e um, sete e 15 dias após o desmame DApD, em amostras tratadas com LPS de E. coli; amostras tratadas com dexametasona e LPS (DxLPS); e amostras não tratadas - grupo controle - CTL. **Diferença significativa (valor $\mathrm{p}=0,0006$ ).

capacidade imunológica mediada por células. Com a supressão da expressão do TNF- $\alpha$, podemos concluir que os mediadores neuroendócrinos desencadeados pelo estresse durante o desmamem abrupto podem causar supressão da resposta imune mediada por células em potros, conforme descrito por diversos autores em outras espécies (Agarwal \& Marshall Junior, 1998; Elenkov et al., 2000; Kiecolt-Glaser et al., 2002).

\section{Conclusão}

O desmame gera uma situação de estresse agudo nos potros, capaz de suprimir a resposta imune mediada por células, evidenciada pela redução da expressão do TNF- $\alpha$ no período de até sete dias após o desmame.

\section{Referências}

Adams, A. A., \& Horohov, D. W. (2013). The effect of an immunomodulator (parapoxvirus ovis) on cell-mediated immunity (CMI) in abruptly weaned foals. Veterinary Immunology and Immunopathology, 153(1-2), 118-122. http://dx.doi.org/10.1016/j.vetimm.2012.11.020. PMid:23312290.

Agarwal, S. K., \& Marshall Junior, G. D. (1998). Glucocorticoid-induced type 1/type 2 cytokine alterations in humans: a model for stress-related immune dysfunction. Journal of Interferon \& Cytokine Research, 18(12), 1059-1068. http://dx.doi.org/10.1089/jir.1998.18.1059. PMid:9877450.

Bemelmans, M. H., Van Tits, L. J., \& Buurman, W. A. (1996). Tumor necrosis factor: function, release, and clearance. Critical Reviews in Immunology, 16(1), 1-11. http://dx.doi.org/10.1615/CritRevImmunol.v16.i1.10. PMid:8809470.

Chrousos, G. P. (1995). The hypothalamic-pituitary-adrenal axis and immune-mediated inflammation. The New England Journal of Medicine, 332(20),1351-1362. http://dx.doi.org/10.1056/NEJM199505183322008. PMid:7715646.

Cywinska, A., Szarska, E., Degorski, A., Guzera, M., Gorecka, R., Strzelec, K., Kowalik, S., Schollenberger, A., \& Winnicka, A. (2013). Blood phagocyte activity after race training sessions in Thoroughbred and Arabian horses. Research in Veterinary Science, 95(2), 459-464. http://dx.doi.org/10.1016/j.rvsc.2013.04.020. PMid:23664016.

DeRijk, R., Michelson, D., Karp, B., Petrides, J., Galliven, E., Deuster, P., Paciotti, G., Gold, P. W., \& Sternberg, E. M. (1997). Exercise and circadian rhythm-induced variations in plasma cortisol differentially regulate interleukin- $1 \beta$ (IL-1 $\beta$ ), IL-6 and tumor necrosis factor- $\alpha$ (TNF- $\alpha$ ) production in humans: high sensitivity of TNF- $\alpha$ and resistance of IL-6. The Journal of Clinical Endocrinology and Metabolism, 82(7), 2182-2191. PMid:9215292.

Elenkov, I. J., Chrousos, G. P., \& Wilder, R. L. (2000). Neuroendocrine regulation of IL-12 and TNF- [alpha]/ IL-10 balance: clinical implications. Annals of the New York Academy of Sciences, 917(1), 94-105. http://dx.doi. org/10.1111/j.1749-6632.2000.tb05374.x. PMid:11268424. 
Harbuz, M. S., \& Lightman, S. L. (1992). Stress and the hypothalamic-pituitary-adrenal axis: acute, chronic and immunological activation. The Journal of Endocrinology, 134(3), 327-339. http://dx.doi.org/10.1677/joe.0.1340327. PMid:1402543.

Hoffman, R. M., Kronfeld, D. S., Holland, J. L., \& Greiwe-Crandell, K. M. (1995). Preweaning diet and stall weaning method influences on stress response in foals. Journal of Animal Science, 73(10), 2922-2930. http://dx.doi. org/10.2527/1995.73102922x. PMid:8617662.

Houpt, K. A., Hintz, H. F., \& Butler, W. R. (1984). A preliminary study of two methods of weaning foals. Applied Animal Behaviour Science, 12(1-2), 177-181. http://dx.doi.org/10.1016/0168-1591(84)90107-2.

Juul-Madsen, H. R., Jensen, K. H., Nielsen, J., \& Damgaard, B. M. (2010). Ontogeny and characterization of blood leukocyte subsets and serum proteins in piglets before and after weaning. Veterinary Immunology and Immunopathology, 133(2-4), 95-108. http://dx.doi.org/10.1016/j.vetimm.2009.07.006. PMid:19699538.

Kiecolt-Glaser, J. K., Mcguire, L., Robles, T. F., \& Glaser, R. (2002). Psychoneuroimmunology: psychological influences on immune function and health. Journal of Consulting and Clinical Psychology, 70(3), 537-547. http://dx.doi.org/10.1037/0022-006X.70.3.537. PMid:12090368.

Kim, Y. K., \& Maes, M. (2003). The role of the cytokine network in psychological stress. Acta Neuropsychiatrica, 15(3), 148-155. http://dx.doi.org/10.1034/j.1601-5215.2003.00026.x. PMid:26983358.

Malinowski, K., Hallquist, N. A., Helyar, L., Sherman, A. R., \& Scanes, C. G.G. (1990). Effect of different separation protocols between mares and foals on plasma cortisol and cell-mediated immune response. Journal of Equine Veterinary Science, 10(5), 363-368. http://dx.doi.org/10.1016/S0737-0806(06)80098-5.

Mills, D. S., \& Nankervis, K. (2005). Comportamento eqüino (p. 213). São Paulo: Roca.

Morel, M. D. (2003). Equine reproductive physiology, breeding and stud management (2. ed., p. 384). New York: Cabi Publishing.

Morel, M. D. (2005). Breeding horses (p. 224). Oxford: Blackwell Publishing. http://dx.doi.org/10.1002/9780470751176.

Page, A. E., Loynachan, A. T., Bryant, U., Stills Junior, H. F., Adams, A. A., Gebhart, C. J., Pusterla, N., \& Horohov, D. W. (2011). Characterization of the interferon $\gamma$ response to Lawsonia intra-cellularis using an equine proliferative enteropathy challenge (EPE). Veterinary Immunology and Immunopathology, 143(1-2), 55-65. http://dx.doi.org/10.1016/j.vetimm.2011.06.023. PMid:21719114.

Schweizer, C. M., Cable, C., \& Squires, E. L. (2006). Breeder's guide to mare, foal \& stallion care: the horse health care library (p. 382). Kentucky: Eclipse Press/The Horse Health Care Library.

Stull, C. L., Spier, S. J., Aldridge, B. M., Blanchard, M., \& Stott, J. L. (2004). Immunological response to long-term transport stress in mature horses and effects of adaptogenic dietary supplementation as an immunomodulator. Equine Veterinary Journal, 36(7), 583-589. http://dx.doi.org/10.2746/0425164044864589. PMid:15581322.

Turner, J. L., Arns, M. J., Minton, J. E., \& Pruitt, J. A. (2003). Effects of abrupt VS. gradual weaning of cortisol and immune function responses of foals. The Professional Animal Scientist, 19(1), 55-61. http://dx.doi.org/10.15232/ S1080-7446(15)31376-0. 